\title{
Effect of Diabetes Mellitus on Outcomes of Colorectal Cancer
}

\author{
Geum Youb Noh, Dae-Yong Hwang ${ }^{1}$, Yoon Hee Choi ${ }^{2}$, Yun Yong Lee \\ Department of Internal Medicine, Korea Cancer Center Hospital, Seoul, \\ ${ }^{1}$ Department of Surgery, Konkuk University Medical Center, Konkuk University School of Medicine, Seoul, \\ ${ }^{2}$ Department of Internal Medicine, Dongnam Institue of Radiological and Medical Sciences, Busan, Korea
}

Purpose: Many studies have revealed that diabetes mellitus (DM) increases a person's lifetime risk of colorectal cancer and that DM is associated with a worse outcome of colon cancer, but this association is controversial. In this study, we intended to examine the relationship between DM and the long-term outcomes of colorectal cancer.

Methods: A retrospective analysis was conducted on 657 patients who underwent surgery due to colorectal cancer between 1997 and 2004 at Korea Cancer Center Hospital. The operations were done by a single surgeon. With a median follow-up of 4.7 years, we analyzed differences in recurrence-free survival (RFS) and overall survival (OS) between patients with DM and those without DM.

Results: Of the 657 patients, 374 (57\%) were males and 67 (10\%) had DM. There was no difference in age at diagnosis, sex and pathologic stage of colorectal cancer according to the presence of DM. There were no difference in the RFS and the OS of colon cancer between the patients with DM and those without DM. At 5 years, the RFS was $71.3 \%$ in diabetic patients vs. $70.4 \%$ in non-diabetic patients $(\mathrm{P}=0.480)$, and the $\mathrm{OS}$ was $68.8 \%$ in diabetic patients vs. $75.0 \%$ in non-diabetic patients $(\mathrm{P}=0.498)$. There was no difference in the median survival between the groups $(9.6$ years in the diabetic group vs. 10.6 years in the non-diabetic group; $\mathrm{P}=0.495)$.

Conclusion: In this study, we did not find any relation between the presence of DM and either the recurrence or the survival in cases of colorectal cancer. More studies to elucidate whether the influence of DM is directly related to a higher rate of cancer recurrence or survival are needed.

Keywords: Diabetes mellitus; Colorectal neoplasms; Survival; Recurrence

\section{INTRODUCTION}

The prevalence of diabetes mellitus $(\mathrm{DM})$ is rapidly increasing worldwide. During the last 30 years in Korea, the prevalence of DM has become 5-6 times higher increasing from $1.5 \%$ to approximately $10 \%$ [1-3].This increase is much higher than that of other developed countries such as the US.

Received: July 20, 2010 Accepted: September 27, 2010

Correspondence to: Yun Yong Lee, M.D.

Department of Internal Medicine, Korea Cancer Center Hospital, 75

Nowon-gil, Nowon-gu, Seoul 139-706, Korea

Tel: +82-2-970-2210, Fax: +82-2-970-2456

Email: ylee54@kcch.re.kr

(C) 2010 The Korean Society of Coloproctology

This is an open-access article distributed under the terms of the Creative Commons Attribution NonCommercial License (http://creativecommons.org/licenses/by-nc/3.0) which permits unrestricted non-

commercial use, distribution, and reproduction in any medium, provided the original work is properly cited
Many studies have reported that type $2 \mathrm{DM}$ is related to the risk of developing cancer. Many studies of meta analysis revealed that a history of DM significantly increased the incidence of liver, pancreatic, colorectal, renal, bladder, endometrial, and breast cancer [4-10]. A ten-year prospective cohort study of $1,298,385$ Koreans who received health insurance from the National Health Insurance Corporation showed elevated fasting serum glucose levels and a diagnosis of DM to be independent risk factors for several major cancers, and the risk tended to increase with increasing level of fasting serum glucose [11].

The association between DM and colorectal cancer has been studied most actively. Many reports demonstrated that the risk of colorectal cancer was increased in DM patients [6, 12]. In addition, DM affected the prognosis for colorectal cancer. Coughlin et al. [13] reported that the mortality rate for colorectal cancer was higher in patients with DM, and a study by Meyerhardt et al. [14] reported that the five-year survival rate for colorectal 
cancer was reduced in DM patients.

As the prevalence of DM increases, the prevalence of colorectal cancer with DM will increase. If DM were to be found to affect the prognosis for colorectal cancer, it would be a serious health issue. To determine the effect of DM on the outcomes for colorectal cancer, we conducted a retrospective analysis of colorectal cancer patients who underwent surgery.

\section{METHODS}

This study analyzed retrospectively the data on 657 colorectal cancer patients who underwent surgery by a single surgeon at Korea Cancer Center Hospital from 1997 to 2004. All patients had a histologically confirmed adenocarcinoma, and the mean follow-up duration was 4.7 years. Based on the medical records, the existence of DM, age at diagnosis of colorectal cancer, gender, stage at diagnosis, histological differentiation, recurrence, and survival were examined. Patients that either had a history of DM or were taking medications for DM were classified as DM patients.

The survival rate and the recurrence rate were analyzed according to the existence of DM. Overall survival (OS) was defined as the time from study entry to death from any cause.

Table 1. Baseline characteristics for 657 patients with colorectal cancer

\begin{tabular}{lccc}
\hline Variable & $\begin{array}{c}\text { No DM } \\
(\mathrm{n}=590)\end{array}$ & $\begin{array}{c}\text { DM } \\
(\mathrm{n}=67)\end{array}$ & P-value \\
\hline Age & $57.6 \pm 10.3$ & $61.2 \pm 11.2$ & 0.010 \\
Gender (M:F) & $332: 258$ & $42: 25$ & 0.303 \\
BMl (kg/m²) & $23.2 \pm 3.1$ & $24.9 \pm 3.7$ & 0.001 \\
T stage (depth) & & $2(3.4)$ & 0.285 \\
T1 & $28(5.3)$ & $6(10.3)$ & \\
T2 & $79(15)$ & $39(67.2)$ & \\
T3 & $284(53.9)$ & $11(19.0)$ & \\
T4 & $136(25.8)$ & & \\
N stage (positive LN) & & & \\
N0 (0 node) & $306(58.1)$ & $36(62.1)$ & \\
N1 (1-3 nodes) & $140(26.6)$ & $15(25.9)$ & \\
N2 (> 4 nodes) & $81(15.4)$ & $7(12.1)$ & \\
Stage & & & 0.767 \\
I & $85(14.4)$ & $7(10.4)$ & \\
II & $250(42.4)$ & $32(47.8)$ & \\
III & $220(37.3)$ & $26(38.8)$ & \\
IV & $35(5.9)$ & $2(3.0)$ & \\
Grade & & & 0.678 \\
Well & $183(31)$ & $19(28.4)$ & \\
Moderate & $371(62.9)$ & $44(65.7)$ & \\
Poor & $23(3.9)$ & $4(6.0)$ & \\
Other & $13(2.2)$ & $0(0)$ & \\
\hline
\end{tabular}

Values are presented as mean \pm SD or number (\%).

DM, diabetes mellitus; BMI, body mass index; LN, lymph node.
Recurrence-free survival (RFS) was defined as the time from study entry to tumor recurrence or occurrence of a new primary colon cancer. For RFS, patients who died without known recurrence were selected at their final documented evaluation. Statistical analysis was conducted with SPSS ver.12.0 (SPSS Inc., Chicago, IL, USA), and a P-value of less than 0.05 was considered to be statistically significant. The distribution of baseline characteristics between DM and non-DM patients was evaluated using either Fisher's exact or $\chi^{2}$ tests in the case of categorical variables or student's t-test in the case of continuous variables. The OS and the RFS were examined using the Kaplan-Meier method and a Cox proportional hazards regression analysis.

\section{RESULTS}

Of the 657 patients included in our analysis, 67 (10\%) had DM. The clinical characteristics of the subjects according to DM are presented in Table 1. The mean age of the DM group was higher than that of non-DM group $(61.2 \pm 11.2$ years and $57.6 \pm 10.3$ years, respectively). Among the subjects, 374 (57\%) were male, and 283 were female (43\%), and there was no significant difference in gender according to DM. The body mass index (BMI) was significantly higher in the DM group than in the non-DM group $\left(24.9 \pm 3.7 \mathrm{~kg} / \mathrm{m}^{2}\right.$ and $23.2 \pm 3.1 \mathrm{~kg} / \mathrm{m}^{2}$, respectively). In addition, for the patients with a BMI of over $25 \mathrm{~kg} / \mathrm{m}^{2}$, the prevalence of DM was $16.9 \%$, which was significantly higher than the $7.1 \%$ for those with a BMI of less than $25 \mathrm{~kg} / \mathrm{m}^{2}(\mathrm{P}<0.001)$. The stage at diagnosis of colorectal cancer and the histological differentiation of colorectal cancer were not different between the DM and the non-DM groups.

The survival rate and the recurrence rate were analyzed according to presence or absence of DM. Kaplan-Meier curves presenting the OS and the RFS are shown in Figs. 1, 2, respec-

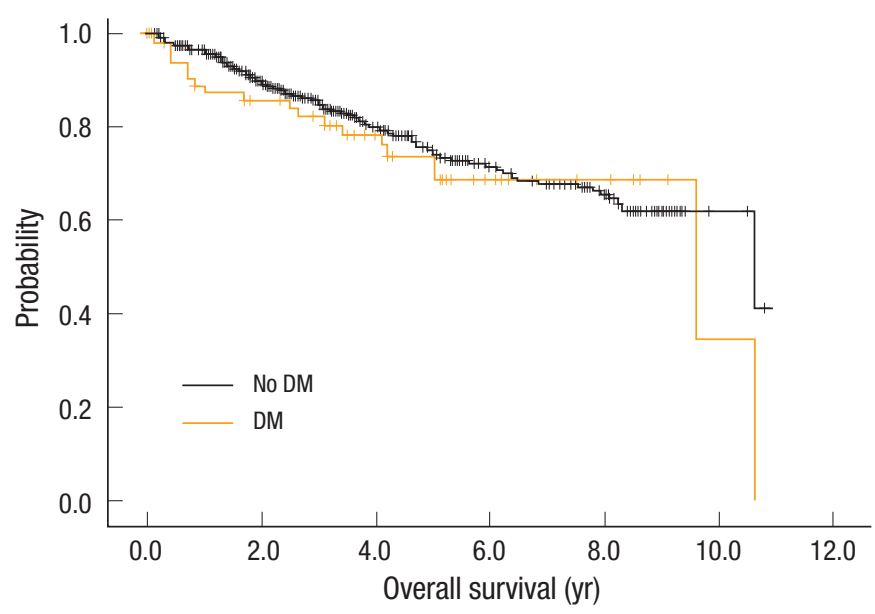

Fig. 1. Overall survival for patients with colorectal cancer by diabetes mellitus (DM) status. 
tively. The five-year survival rate in the DM group was 68.0\% and that in the non-DM group was $75 \%$, the difference not being statistically significant $(\mathrm{P}=0.498)$. The five-year $\mathrm{RFS}$ rate in the DM group was $64.0 \%$ and that in the non-DM group was $69.0 \%$, the difference not being statistically significant $(\mathrm{P}=0.954)$. In the Cox proportional hazard regression analysis, we examined the influence of DM after adjustment for age and BMI. In the non-DM group, in comparison to the DM group, the risk of recurrence was lower by $6.2 \%$, and death was lower by $25 \%$, but the difference was not significant (Table 2). Moreover, when we compared the mean survival of the DM and the non-DM groups, they were not significantly different (9.6 years and 10.6 years, respectively, $\mathrm{P}=0.495$ ).

\section{DISCUSSION}

This study showed that the recurrence rate and the survival rate for colorectal cancer after surgery were not significantly different according to the presence or absence of DM. In addition, we could not find any significant correlation between DM and the occurrence of colorectal cancer when we consider that the prevalence of DM among the colorectal cancer patients in this study was almost the same prevalence as in the general Korean population during the same period [1-3]. Many studies reported

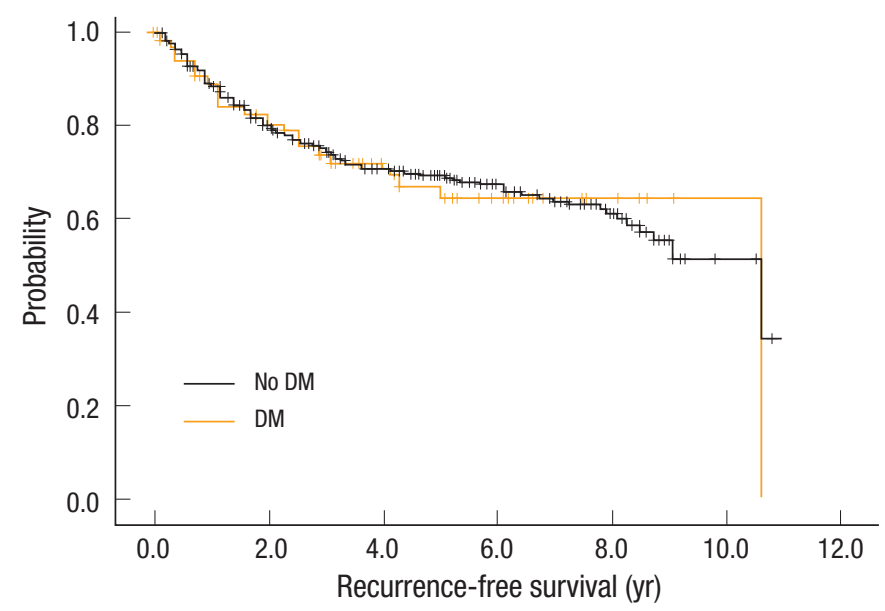

Fig. 2. Recurrence-free survival for patients with colorectal cancer by diabetes mellitus (DM) status. an increased risk of DM patients developing colorectal cancer. The suggested mechanisms were hyperinsulinemia caused by insulin resistance, increase of insulin-like growth factor (IGF), changes of adipocytokines, chronic inflammation and increased oxidative stress in DM patients.

IGF-1 is an important hormone for growth and development, and leads to cell division and cell transformation. IGF binding protein (IGFBP) inhibits its roles by binding with IGF. In hyperinsulinemia, insulin is known to bind directly to the IGF-1 receptor or to work in competition with IGFBP to increase the free IGF-1 level in the blood and eventually to be able to induce cancer by inhibiting apoptosis and promoting cell proliferation $[15,16]$. In addition, slower bowel transit time and elevated fecal bile acid concentrations frequently observed in DM allow more exposure to toxic materials, thus $\mathrm{p}$ provoke colorectal cancer [17]. Moreover, when the blood sugar level is not well-controlled, oxidative stress is increased, and this leads to a pro-inflammatory condition. Reactive oxygen species (ROS) are known to damage cellular DNA and to promote cancer in various steps by reacting with proteins or lipids in cells [18].

There are several reports that the prognosis for colorectal cancer is poor in DM patients. In a study conducted with 3,759 surgically treated colorectal cancer patients, the five-year disease-free survival and OS rates were significantly decreased, even after adjustments for age, gender, race, and stage, location and histological differentiation of tumors [14]. Meta analyses on the mortality rate, recurrence rate and treatment-related complications of colorectal cancer [19] revealed that peri-operative short-term mortality rates were higher among DM patients; the long-term mortality rate was also increased by $32 \%$ in DM patients. Limited use of anticancer drugs because of complications of DM, such as cardiovascular disease and renal and nervous disease, and higher risk of infection after anti-cancer therapy could explain the high mortality rate in DM patients. However, when we consider Meyerhardt et al. [14] report that the recurrence rate of cancer was also higher in DM patients, we should also consider that the increased recurrence caused by DM may have led to the increased mortality.

However, other studies did not find any correlation between $\mathrm{DM}$ and the incidence of or the prognosis for colorectal cancer. An epidemiological study of Kune et al. [20] reported that the prevalence of DM in colorectal cancer patients was the same

Table 2. Hazard ratio of diabetes in colorectal cancer

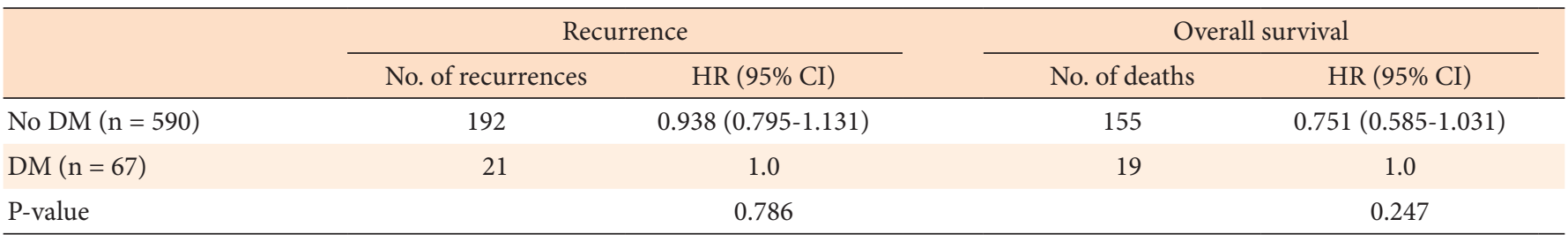

HR, hazard ratio; CI, confidence interval; DM, diabetes mellitus. 
as that of the control group. Another epidemiological study by Will et al. [21], which included 1 million persons, reported that the risk of colorectal cancer was higher in DM patients, but there was no difference in mortality rate according to DM. In addition, a study by Shonka et al. [22] did not show any significant correlation between DM and the prognosis for colorectal cancer. Also, our study did not observe any correlation between colorectal cancer and DM. One reason that the correlation between $\mathrm{DM}$ and colorectal cancer is different may be various DM-related factors, such as obesity, therapeutic agents and blood sugar control, not having been controlled properly. Second reason may be due to the different criteria used in each study to define the existence of DM. As most studies defined the existence of DM according to reports of patients instead of applying glucose tolerance test, there could be DM patients who were not classified as DM patients. Another reason may be hyperinsulinemia due to insulin resistance being maintained only in the early stage of DM. With the progress of DM, hypoinsulinaemia is observed because of islet-cell dysfunction. The negative effect of hyperinsulinemia on the prognosis for colorectal cancer could diminish.

As limitations of this study, the number of subjects is relatively small, and the analysis was retrospective. Moreover, because we defined the existence of DM only as a medical history DM or a history of medications for DM in the medical record, some actual DM patients might not have been included in the DM group. However, this study has a strong point in that the subjects underwent surgery by a single surgeon at a single center so that the difference in complications and prognosis caused by the difference in surgical techniques could be excluded.

Considering that the prevalence of DM has increased greatly, many DM patients are not diagnosed, and the risk of cancer increases due to longer life expectancy, the increase in the occurrence of and the mortality from colorectal cancer in DM patients is expected to become a very important issue of public health. More large-scaled prospective studies are necessary in the future, and more efforts to use exact diagnostic criteria of DM in a study are needed. In addition, investigating the prognosis of colorectal cancer according to blood sugar control is thought to be clinically important, and measuring the blood insulin levels of the subjects may be helpful in elucidating the mechanism of colorectal cancer development in DM patients.

This study to investigate the effect of DM on the prognosis for colorectal cancer did not show any differences in the stage at diagnosis, the survival rate and the recurrence rate of colorectal cancer according to the existence or the absence of DM. More large-scaled studies are necessary in the future.

\section{CONFLICT OF INTEREST}

No potential conflict of interest relevant to this article was reported.

\section{REFERENCES}

1. Kim SG, Choi DS. The present state of diabetes mellitus in Korea. J Korean Med Assoc 2008;51:791-8.

2. Cho NH. Prevalence of diabetes and management status in Korean population. Korean J Med 2005;68:1-3.

3. Kim SA, Park WS, Ohrr HC, Kang HY, Lee DH, Yi SW, et al. Prevalence and management status of diabetes mellitus in Korea. Korean J Med 2005;68:10-7.

4. El-Serag HB, Hampel H, Javadi F. The association between diabetes and hepatocellular carcinoma: a systematic review of epidemiologic evidence. Clin Gastroenterol Hepatol 2006;4:369-80.

5. Huxley R, Ansary-Moghaddam A, Berrington de González A, Barzi F, Woodward M. Type-II diabetes and pancreatic cancer: a meta-analysis of 36 studies. Br J Cancer 2005;92: 2076-83.

6. Larsson SC, Orsini N, Wolk A. Diabetes mellitus and risk of colorectal cancer: a meta-analysis. J Natl Cancer Inst 2005;97: 1679-87.

7. Washio M, Mori M, Khan M, Sakauchi F, Watanabe Y, Ozasa K, et al. Diabetes mellitus and kidney cancer risk: the results of Japan Collaborative Cohort Study for Evaluation of Cancer Risk (JACC Study). Int J Urol 2007;14:393-7.

8. Larsson SC, Orsini N, Brismar K, Wolk A. Diabetes mellitus and risk of bladder cancer: a meta-analysis. Diabetologia 2006;49: 2819-23.

9. Friberg E, Orsini N, Mantzoros CS, Wolk A. Diabetes mellitus and risk of endometrial cancer: a meta-analysis. Diabetologia 2007;50:1365-74.

10. Larsson SC, Mantzoros CS, Wolk A. Diabetes mellitus and risk of breast cancer: a meta-analysis. Int J Cancer 2007;121:856-62.

11. Jee SH, Ohrr H, Sull JW, Yun JE, Ji M, Samet JM. Fasting serum glucose level and cancer risk in Korean men and women. JAMA 2005;293:194-202.

12. Khaw KT, Wareham N, Bingham S, Luben R, Welch A, Day N. Preliminary communication: glycated hemoglobin, diabetes, and incident colorectal cancer in men and women: a prospective analysis from the European prospective investigation into cancer-Norfolk study. Cancer Epidemiol Biomarkers Prev 2004;13:915-9.

13. Coughlin SS, Calle EE, Teras LR, Petrelli J, Thun MJ. Diabetes mellitus as a predictor of cancer mortality in a large cohort of US adults. Am J Epidemiol 2004;159:1160-7.

14. Meyerhardt JA, Catalano PJ, Haller DG, Mayer RJ, Macdonald JS, Benson AB 3rd, et al. Impact of diabetes mellitus on outcomes in patients with colon cancer. J Clin Oncol 2003;21:433-40.

15. Giovannucci E, Ascherio A, Rimm EB, Colditz GA, Stampfer MJ, Willett WC. Physical activity, obesity, and risk for colon cancer and adenoma in men. Ann Intern Med 1995;122:327-34.

16. Frezza EE, Wachtel MS, Chiriva-Internati M. Influence of obesity on the risk of developing colon cancer. Gut 2006;55:285-91.

17. Stadler J, Yeung KS, Furrer R, Marcon N, Himal HS, Bruce WR. Proliferative activity of rectal mucosa and soluble fecal bile acids in patients with normal colons and in patients with colonic pol- 


\section{Coloproctology Geum Youb Noh, et al.}

yps or cancer. Cancer Lett 1988;38:315-20.

18. Federico A, Morgillo F, Tuccillo C, Ciardiello F, Loguercio C. Chronic inflammation and oxidative stress in human carcinogenesis. Int J Cancer 2007;121:2381-6.

19. Stein KB, Snyder CF, Barone BB, Yeh HC, Peairs KS, Derr RL, et al. Colorectal cancer outcomes, recurrence, and complications in persons with and without diabetes mellitus: a systematic review and meta-analysis. Dig Dis Sci 2010;55:1839-51.

20. Kune GA, Kune S, Watson LF. Colorectal cancer risk, chronic ill- nesses, operations and medications: case control results from the Melbourne Colorectal Cancer Study 1988. Int J Epidemiol 2007; 36:951-7.

21. Will JC, Galuska DA, Vinicor F, Calle EE. Colorectal cancer: another complication of diabetes mellitus? Am J Epidemiol 1998; 147:816-25.

22. Shonka NA, Anderson JR, Panwalkar AW, Reed EC, Steen PD, Ganti AK. Effect of diabetes mellitus on the epidemiology and outcomes of colon cancer. Med Oncol 2006;23:515-9. 